Review

\title{
Insights into the Nanog gene: A propeller for stemness in primitive stem cells
}

\author{
Wei Zhang ${ }^{1 *}$, Yi Sui ${ }^{2,3^{*}}$, Jun $\mathrm{Ni}^{4}$, Tao Yang ${ }^{\star} \bowtie$ \\ 1. Department of Pharmacology, School of Basic Medicine, Hebei University of Chinese Medicine, Shijiazhuang 050200, China; \\ 2. Center for Diabetic Systems Medicine, Guangxi Key Laboratory of Excellence, Guilin Medical University, Guilin 541004, China; \\ 3. Department of Gynaecology \& Obstetrics, The Chinese University of Hong Kong, Shatin, N.T., Hong Kong, China; \\ 4. Research Center for Translational Medicine, Shanghai East Hospital, Tongji University School of Medicine, Shanghai 200120, China. \\ "These two authors contributed equally to this work.
}

$\triangle$ Corresponding author: Dr. Tao Yang, Research Center for Translational Medicine, Shanghai East Hospital, Tongji University School of Medicine, 150 Jimo Road, Shanghai 200120, China. Phone: 86-21-61569714; Fax: 86-21-33923060; E-mail: taoyang@tongji.edu.cn

(c) Ivyspring International Publisher. Reproduction is permitted for personal, noncommercial use, provided that the article is in whole, unmodified, and properly cited. See http://ivyspring.com/terms for terms and conditions.

Received: 2016.06.01; Accepted: 2016.09.04; Published: 2016.10.25

\begin{abstract}
Self-renewal and pluripotency are two major characteristics of embryonic stem cells (ESCs) that allow ESCs to maintain stem cell population, and differentiate into multiple types of adult tissues. Nanog is the key transcription factor that controls both self-renewal and pluripotency of ESCs. Similarly, cancer stem cells (CSCs) are capable of preserving population and initiating new tumor development by self-renewal. Expression of Nanog family proteins can be increased in many types of cancer which is correlated with tumor outcomes. In this review we summarized the recent understanding of the roles and mechanisms of Nanog in ESC regulation under physiological conditions. In addition, we describe the function of Nanog family proteins in different types of cancer, and the association of Nanog with clinical outcomes. Taken together, Nanog proteins are central regulators controlling both ESCs and CSCs, and are considered as a prognostic marker in many types of cancer. These findings supported the possibility of novel therapeutic potentials of Nanog against cancers.
\end{abstract}

Key words: Nanog, embryonic stem cell, pluripotency, cancer stem cell, metastasis, cancer recurrence

\section{Introduction}

Self-renewal and pluripotency are two distinctive characteristics of undifferentiated embryonic stem cells (ESCs), which enable ESCs to keep their own stemness and to differentiate into multiple lineages. Any error of the tightly-regulated self-renewal and pluripotency processes may lead to developmental defects. Nanog is a critical transcription factor which maintains both self-renewal and pluripotency of ESCs ${ }^{1,2}$. Nanog is selectively expressed in undifferentiated pluripotent cells but not in adult cells. The expression of Nanog is accurately controlled during stem cell differentiation. Nanog is an upstream regulator of STAT3 and Oct4 in maintaining ESC clonal expansion ${ }^{2}$, as well as the leukemia inhibitory factor (LIF)/STAT3 complex ${ }^{1}$. It has been reported that Nanog can regulate pluripotency and cell differentiation through the interaction with complexes formed by transcription factors including Oct4, Sox2, Klf4 and Slug 3,4. Nanog is involved in the pathways which form a reciprocal regulatory circuit to balance self-renewal, proliferation, and differentiation of ESCs. In addition, Nanog can reduce gene transcriptional noises by regulating polycomb complexes, DNA methylation, expression of microRNAs, so that most stochastic and aberrant induction of differentiation can be prevented $^{5-7}$.

Nanog protein, coded by Nanog1 gene, consists of 305 amino acids and contains conserved homeodomain that binds to DNA. Human Nanog1 gene (gi 13376297) is localized on chromosome 12 and consists of 4 exons and 3 introns with a 915 bp open 
reading frame $(\mathrm{ORF})^{8}$ (Fig. 1). It is very unique that Nanog1 gene has been tandem duplicated into a variant gene Nanog2 (NanogP1), as well as many pseudogenes (NanogP2-P11) during evolution9,10. The comparison of human and chimpanzee genome sequences has revealed that Nanog2 retained its intronic sequences, while NanogP2 to P11 are dispersed, intronless and reversely transcribed integrants ${ }^{11}$. Among those pseudogenes, Nanog homeobox pseudogene 8 (NanogP8) is the only one that has an intact ORF12,13. Intriguingly, NanogP8, located on chromosome 15, is free of structural defects and has the potential to be functionally expressed (Fig. 1). Nanog1 and NanogP8 have identical 5 '-untranlated regions (UTRs) except the first $\sim 18$-bp, which are unique to each gene (Fig. 1). The two genes also have very similar $3^{\prime}$-UTRs except for the $\sim 20-b p$ sequence in the Nanog1 3'-UTRs (Fig. 1).

Three Nanog proteins were demonstrated to elicit influence on differentiation of human ESCs.
They were Nanog1, Nanog2 and NanogP814. The functional paralogs of Nanog family have been amplified with multi-Nanog PCR, showing that human ESCs express large amounts of Nanog1 and Nanog2. Nanog1 is essential for the self-renewal and pluripotency of ESCs and is also required for the induced pluripotent stem cells (iPSCs) to reach the ground state $^{1}$. Forced expression of Nanog1 is sufficient to maintain the undifferentiated state of ESCs and targeted disruption of Nanog1 results in loss of ESC identity and differentiation toward primitive endoderm ${ }^{1}$. In contrast, NanogP8 protein is expressed in most human cancer cells, and the Nanog protein level generated by NanogP8 is comparable to that produced by Nanog1 in pluripotent cells ${ }^{14}$. Moreover, NanogP8 is as effective as Nanog1 in the reprogramming of human and murine fibroblasts into iPSCs, suggesting NanogP8 can contribute to promote de-differentiation and/or pluripotency of eukaryotic cells ${ }^{1,14}$.
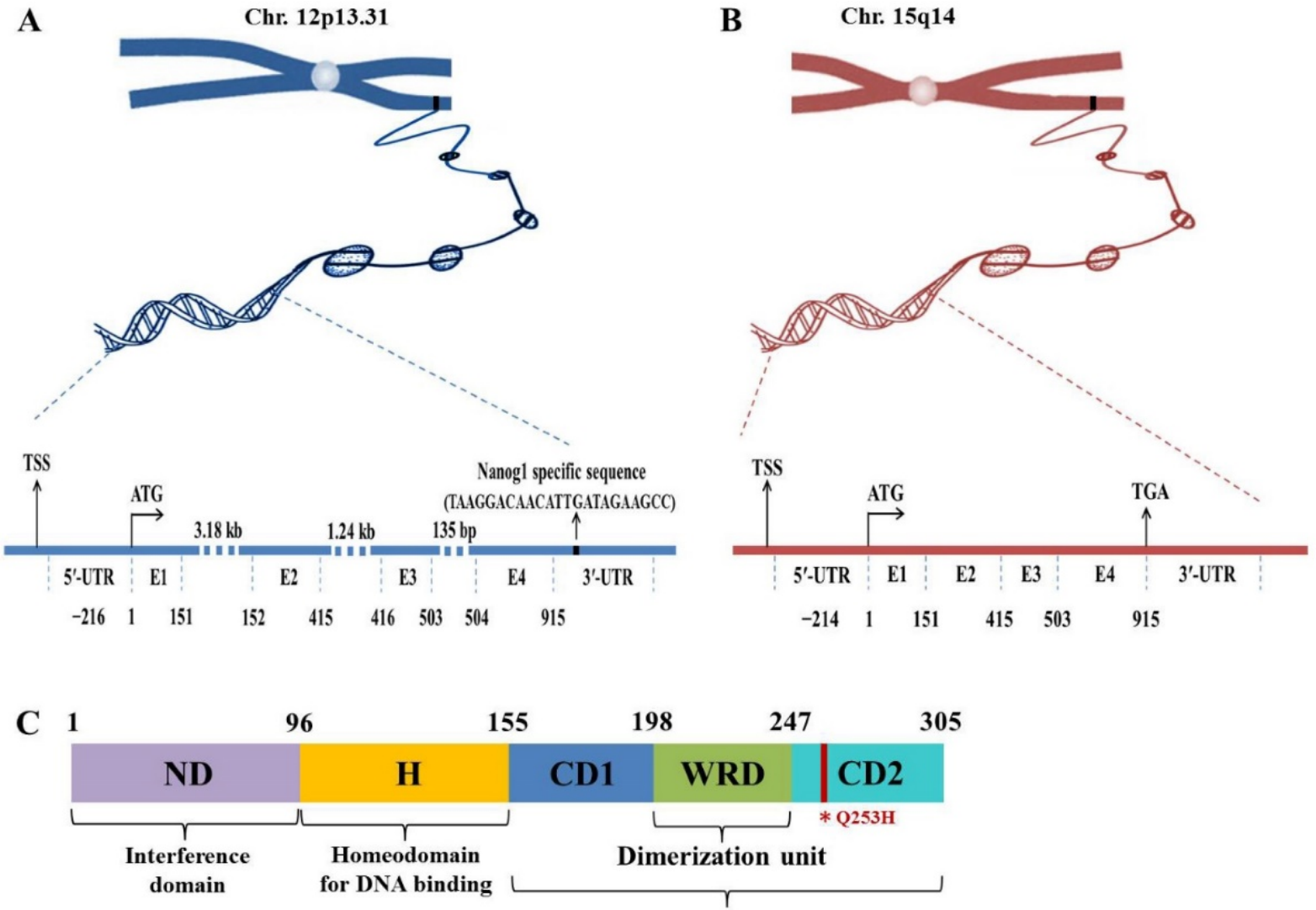

Transactivation domain

Figure 1. Genomic and protein structures of Nanog genes. (A-B) Genomic structures of Nanogl (A), and Nanogp8 gene (B). The 2 genes both have 4 exons (E) with a 915-bp ORF. NanogP8 is a retrotransposed gene and thus lacks introns, whose sizes in Nanogl are indicated. The 2 genes have identical 5'-UTRs except the first $\sim 18$-bp, which are unique to each gene (marked by a green and red rectangle). The 2 genes also have very similar 3 '-UTRs except for the $\sim 20$-bp sequence in the NanogI 3'-UTR (A). The specific sequences in this region were used to design Nanogl/NanogP8-specific PCR primers. TSS, transcriptional start site. (C) Protein structure of Nanogl protein. Nanogl protein consists of an N-terminal "interference" domain (ND), homeodomain (H) important for DNA binding, and a C-terminal domain containing 2 subdomains (CD1 and CD2, responsible for transactivation) and a tryptophan-rich domain (WRD) involved in dimerization. The red line in CD2 indicates the conserved amino acid change $(\mathrm{Q} 253 \mathrm{H})$ in NanogP8 protein. 
Similar to ESCs, cancer stem cells (CSCs) are cancer cells that possess characteristics associated with normal stem cells, including self-renewal and differentiation into multiple cell types. It is hypothesized that CSCs are the one of the major causes of tumor relapse and metastasis by developing new tumor. Therefore, understanding the Nanog-involved mechanism underlying CSC self-renewal and differentiation is essential for developing specific therapy against cancers, especially metastatic cancers. It has been reported that Nanog family members are critical for CSCs: 1) Expression of Nanog proteins is increased in many types of cancer; 2) Enhanced levels of Nanog proteins are related with CSC-like phenotype ${ }^{15,16}$; 3) Knockdown or knockout of Nanog gene could reduce cancer malignancy ${ }^{17-19}$. Altogether, Nanog family proteins are pivotal to maintain the function of ESCs under physiological conditions, as well as CSC phenotype under pathological conditions. In this review we will summarize the recent research progress on Nanog proteins in regulation of both ESCs and CSCs.

\section{The function of Nanog in ESCs}

ESCs are derived from the inner cell mass (ICM) of blastocyst ${ }^{20}$. The expression of Nanog is detectable at embryonic day 6 (E. 6) in proximal epiblast in the region of presumptive primitive streak, and the expression extends distally as the streak elongates during gastrulation and remains restricted to epiblast ${ }^{20}$. Nanog RNA is down-regulated in cells ingressing through the streak to form mesoderm and definitive endoderm ${ }^{20}$. Though LIF and STAT3 are reported to maintain self-renewal of mouse ESCs ${ }^{21}$, LIF/STAT3 is dispensable for maintenance of ICM and human ES cells, and Nanog is identified as a vital regulator to control ESC self-renewal at the upstream of LIF/STAT3, since Nanog-deficient ICM could not generate epiblast and only produce parietal endoderm-like cells ${ }^{1}$. Meanwhile, Nanog-deficient ESCs lost pluripotency and differentiated into extraembryonic endoderm lineage ${ }^{1}$ (Fig. 2).

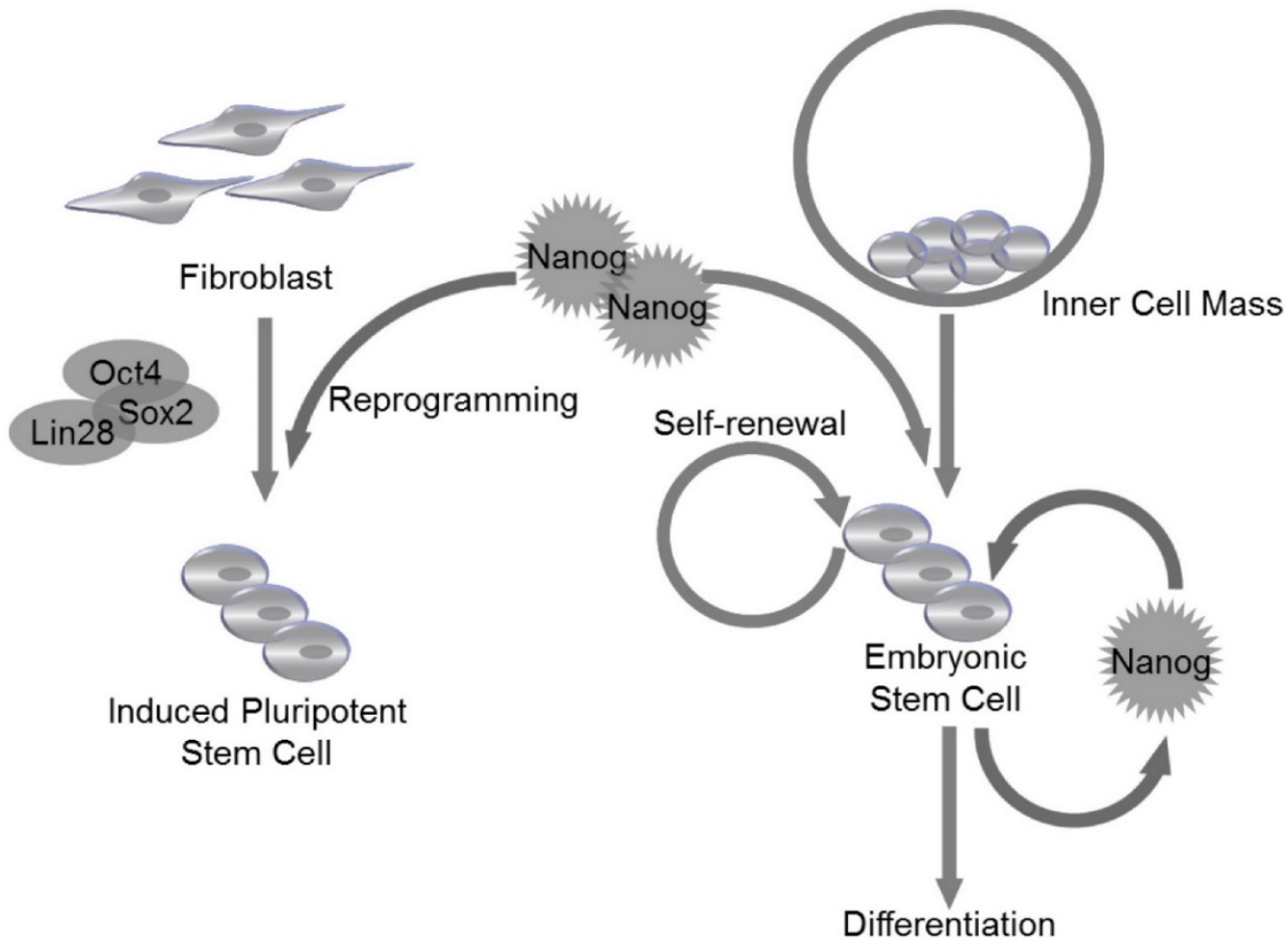

Figure 2. Nanog regulates self-renewal and pluripotency in pluripotent stem cells. Nanog is capable of regulating self-renewal and pluripotency in inner cell mass (ICM)-derived embryonic stem cells (ESCs). Nanog, in combination with Oct4, Sox2 and Lin28, can be utilized to induce efficient reprogramming in fibroblasts to generate induced pluripotent stem cells (iPSCs). 
Nanog protein can be divided into N-terminal and C-terminal halves. The N-terminal half contains a DNA-binding homeodomain and an N-terminal domain. The C-terminal half contains a dimerization domain referred to as the tryptophan repeat, in which every fifth residue is a tryptophan, separating C-terminal domain 1 from C-terminal domain $2^{22}$ (Fig.1 C). Homodimerization of Nanog is critical for promoting pluripotency of ESCs, which are maintained by transcription factors that form a highly interconnected protein interaction network surrounding the homeobox protein Nanog23,24. Enforced expression of Nanog in mouse ESCs promotes self-renewal and alleviates their requirement for LIF' ${ }^{1}$ The enforced expression of the Nanog dimer, but not the monomer, functionally sustains LIF-independent self-renewal of ESCs ${ }^{25}$ (Fig. 2). It is demonstrated that Nanog-Nanog homodimerization is critical for its function in promoting stem cell pluripotency. Another transcription factor Oct4 has been found to interact with Nanog and associate with proteins from multiple repression complexes, including the NuRD, Sin3A and Pml complexes ${ }^{26,27}$. In addition, Nanog, Oct4 and repressor proteins could co-occupy Nanog-target genes in mouse ESCs, suggesting that Nanog and Oct4 may collectively communicate with distinct repression complexes to control gene transcription. Moreover, ESC differentiation could be controlled by Nanog and Oct4 through respective unique repressor complexes on their target genes ${ }^{27}$.

In addition to self-renewal and pluripotency, Nanog also regulates cell cycle of ESCs. Previous study has reported that ESC clones with overexpressed Nanog have accelerated S-phase entry ${ }^{28}$. Under physiological conditions, the C-terminal of Nanog was found to bind to regulatory region of CDK6 and CDC25A, which are served as downstream mediators of Nanog-regulated S-phase entry ${ }^{28}$. Nanog family protein, NanogP8, is highly expressed in T-cell acute lymphoblastic leukemia (T-ALL) cells, and knockdown of NanogP8 by small interfering RNA (siRNA) inhibited cell proliferation, reduced self-renewal, promoted apoptosis and arrested the cell cycle through a p53-mediated pathway ${ }^{29}$. Similarly, in breast cancer cell line, downregulation of Nanog reduced expression level of cyclin E and STAT3, and caused cell arrest at G0/G1 phase $^{30}$. Since Nanog is essential for cell cycle control of ESCs, its own expression is supposed to be tightly controlled during embryonic development. The molecular mechanism underlying Nanog regulation is still unclear. Recently, Zic3 is demonstrated to act as a direct activator of Nanog1 in ESCs by binding to the promoter region ${ }^{31}$. Furthermore, the activity of a mutant Nanog1 promoter with ablated Oct4/Sox2 binding site is rescued by Zic3 overexpression to nearly wild-type levels, suggesting that activation of Nanog1 promoter by Zic3 is independent of Oct4/Sox 2 binding 31 . The expression of Nanog1 is also controlled at epigenetic level. It is reported that downregulation of TET3, a member of TET family that can convert 5-methylcytosine to 5-hydroxymethylcytosine, resulted in the reduction of 5-hydroxymethylcytosine and thus reduced the level of Nanog1 expression in the blastocysts ${ }^{32}$. TET family proteins are thought to be not only regulators of Nanog1, since Costa et al identified 27 high-confidence protein interaction partners of Nanog1 in mouse ESCs by using enhanced purification techniques and a stringent computational algorithm ${ }^{33}$. Exactly, 19 of the 27 hits are unknown Nanog1 binding proteins, and TET1 is confirmed to directly bind to Nanog1. The physiological association of Nanog1 with TET1 synergistically enhanced the efficiency of reprogramming ${ }^{33}$. Co-expression of Nanog1 and TET1 increases 5-hydroxymethylcytosine levels at the loci of Esrrb and Oct4, resulting in priming of their expression before reprogramming to naive pluripotency ${ }^{33}$ (Fig. 2).

\section{The pro-tumorigenic effects of NanogP8}

As a Nanog1 homolog in cancer cells, NanogP8 is demonstrated to play a key role in regulating CSC properties $^{34}$. Many studies have tried to explore the potential mechanism for pro-tumorigenic effects of NanogP835,36, and found that NanogP8 contributes to CSC properties as evidenced in three perspectives: (i) The pivotal role of NanogP8 in maintaining stemness of CSCs at cancer initiation stage; (ii) The upregulation of NanogP8 mRNA and protein level during cancer progression; (iii) The NanogP8 siRNA-based inhibitory effects observed during cancer development stage.

\section{Nanog could confer CSC properties}

Recent findings explored that stemness factors such as Nanog, Oct4, and Sox 2 could reprogram the somatic cells back to an ESC-like state. Likewise, such stemness factors could also be involved in cancer initiation and progression, since the expression levels of aforementioned stemness factors could be abnormally elevated in several types of CSCs. Notably, cell fate in both ESCs and CSCs could be alternatively determined by Nanog, the activation of which at an inappropriate time could result in generation of CSCs rather than normal pluripotent stem cells or differentiated somatic cells ${ }^{37}$. Study on downstream regulatory pathways of Nanog has indicated that several aspects of cancer development 
could be controlled by Nanog, including tumor cell proliferation, motility, epithelial-mesenchymal transition (EMT), immune evasion, drug resistance, malignancy conversion, as well as communication between cancer cells and the surrounding stroma ${ }^{38}$.

\section{Nanog mRNA and protein were up-regulated during cancer progression}

In recent studies, a functional variant of Nanog has been reported to be expressed in cultured cancer cells and xenograft human primary prostate cancer cells. Nanog mRNA in cancer cells is derived predominantly from the retrogene locus of NanogP8, which is dominantly detected in the nucleus of CSCs. The expression level of NanogP8 protein is higher in patient prostate tumors than matched benign tissues 38,39 . NanogP8 mRNA and protein are also found to be enriched in putative CSCs and cancer progenitor cell populations ${ }^{39}$.

\section{The role of NanogP8 in CSCs is validated by NanogP8 knockdown}

When NanogP8 expression was knocked down with a short hairpin RNA (shRNA) vector, the anchorage independency and tumorigenicity were repressed ${ }^{40}$. Extensive loss-of-function analysis has revealed that tumor development could be inhibited by RNA interference (RNAi)-mediated NanogP8 knockdown, establishing a functional significance for NanogP8 expression in cancer cells. Long-term clonal and clonogenic growth of CSCs is also reduced in NanogP8-shRNA-transduced CSCs ${ }^{39,41}$. Altogether, NanogP8 exhibits a crucial role in promoting CSC stemness, and contributes to tumor initiation and development.

\section{The roles of Nanog proteins in miscellaneous cancers}

The dysregulation of NanogP8 can be involved in tumorigenesis through the influence on the CSC population. Elevated NanogP8 expression is associated with poor clinical outcome in numerous epithelial malignancies. NanogP8 protein is enriched in CSCs; whereas the CSCs pools were reduced following downregulation of NanogP8. NanogP8 has also been considered to promote chemoresistance and EMT $^{34,38,42}$. Many experimental anti-cancer treatments have targeted on the NanogP8 and its regulatory pathway components. Knockdown of Nanogp8 through RNAi and microRNAs has significantly suppressed the tumorigenic and metastatic capacity of miscellaneous cancer cells ${ }^{34,43}$. Chiou et al reported that elevated expression of Nanog and Oct4 was found in lung adenocarcinoma (LAC), and double knockdown of Nanog and Oct4 suppressed the Slug expression, reversed the EMT process and blocked the tumorigenic and metastatic ability, which greatly improved the mean survival time of LAC cell-transplanted immunocompromised mice ${ }^{34}$. Immunohistochemistry analysis demonstrated the presence of Nanog, Oct4 and Slug in high-grade LAC. Also, triple positivity of Nanog/Oct4/Slug could predict a worse prognostic outcome of LAC patients, indicating that manipulation on Nanog/Oct4 signaling may be effective in repressing EMT, tumor initiation and metastasis of $\mathrm{LAC}^{34}$ (Fig. 3). Downregulation of NanogP8 by shRNA in human gastric cancer cell line leads to significant reduction of cell proliferation, invasion and migration capacity of cancer cells. Reduced NanogP8 can also induce cell cycle arrest and apoptosiss ${ }^{43}$. Similar scenarios were observed during NanogP8 inhibition in glioblastoma cells $^{44}$ and breast cancer cells ${ }^{30}$. In MCF-7 cells, a breast cancer cell line, downregulation of NanogP8 reduced expression levels of cyclin E and STAT3, thus repressed cell proliferation, colony formation and migration, and caused cell cycle arrest at G0/G1 phase $^{30}$ (Fig. 3). Consistently, proliferation of breast cancer cells MDA-MB-231 was also significantly repressed by the inhibition of NanogP830. These data suggested that NanogP8 could be the potential target in prospective genetic treatment via siRNA against miscellaneous types of cancer. Meanwhile, the microRNAs were also involved in NanogP8 regulation. The miR-214 was demonstrated to regulate ovarian cancer stem cell (OCSC) properties by targeting p53/Nanog axis ${ }^{23}$. As reported, miR-214 can enhance Nanog expression through a direct repression of p53. OCSC population and self-renewal would be enhanced by enforcing expression of miR-214, suggesting that miR-214 may act as a therapeutic target for ovarian cancer ${ }^{23}$ (Fig. 3).

\section{Prostate cancer}

Previous studies have demonstrated pro-tumorigenic functions of NanogP8 in prostate cancer (PCa) $39,42,45$. NanogP8 ${ }^{+} \mathrm{PCa}$ cells exhibited enhanced clonal growth and tumor regenerative capacity ${ }^{42}$, and induction of NanogP8 by a tetracycline-controlled system in PCa cells promoted drug resistance and tumor regeneration ${ }^{42,45}$. These pro-tumorigenic effects were associated with an upregulation of CXCR4, IGFBP5, CD133 and ALDH1 ${ }^{42}$. A study of CSC maker expression in 133 PCa patients has shown that all patients have positive expression of Nanog and Oct4, and a significant correlation was observed between Nanog and hypoxia-inducible factor (HIF)-1a in those patients ${ }^{46}$. These studies suggest that Nanog may be a promising biomarker for diagnosis of malignant $\mathrm{PCa}$, and 
co-expression of Nanog and HIF-1a may predict an unfavorable prognosis since such co-expression may accelerate carcinogenesis and metastasis of $\mathrm{PCa}^{46}$.

\section{Hepatocellular carcinoma}

The expression of CSC markers, Nanog and Oct4, is examined in 228 hepatocellular carcinoma (HCC) patients, and it has been found that the co-expression of Oct4 and Nanog dramatically associated with tumor size and vascular invasion, and is an independent predictor of postoperative recurrence and poor prognosis ${ }^{47}$. Nanog ${ }^{+}$CSCs isolated from HCC were capable of differentiating into mature cancer cells in vitro and in vivo ${ }^{48}$. These cells also exhibited resistance to therapeutic agents (e.g., sorafenib and cisplatin) and a high capacity for tumor invasion and metastasis ${ }^{48}$. The insulin-like growth factor (IGF) pathway components can function as downstream molecules of Nanog in HCC, since IGF2 and IGF receptors are upregulated in Nanog ${ }^{+}$CSCs and knockdown of Nanog inhibits the expression of IGF1 receptor (IGF1R); whereas overexpression of Nanog in Nanog- CSCs increases the expression of IGF1R ${ }^{48}$ (Fig. 3). However, it remains unclear how these CSCs contribute to HCC initiation and progression. A clue was reported that Nanog promoted EMT process in HCC by activated
Smad3-mediated Snail expression ${ }^{38}$. The co-expression of Nanog and phosphorylated Smad3 may be considered as a potential predictor for poor prognosis in HCC patients. Additionally, HCC cells in the tumor edge area have displayed higher Nanog expression than cells in the tumor center, which might suggest an important role of Nanog in HCC metastasis $^{38}$.

\section{Leukemia}

NanogP8 is expressed in primary T cells isolated from T-ALL patients, and the expression level is correlated with cell proliferation and self-renewal ${ }^{29}$. Knockdown of NanogP8 by RNAi promotes cell apoptosis and cell cycle arrest through a p53-mediated pathway ${ }^{29}$. Nanog1 and Nanog2 protein variants have been identified in human $t(4 ; 11)$ leukemia cells, and they have equivalent functions in activating specific downstream stem cell genes ${ }^{9}$. Properties of leukemia stem cells could be conferred by transcriptional activation of Nanog2. Transcripts of Nanog2, Oct4 and Sox2 have been recently identified in multiple human leukemic cell types, suggesting Nanog, in collaboration with stemness-related transcriptional factors, is also important in regulating leukemic stem cell functions ${ }^{9,29}$.

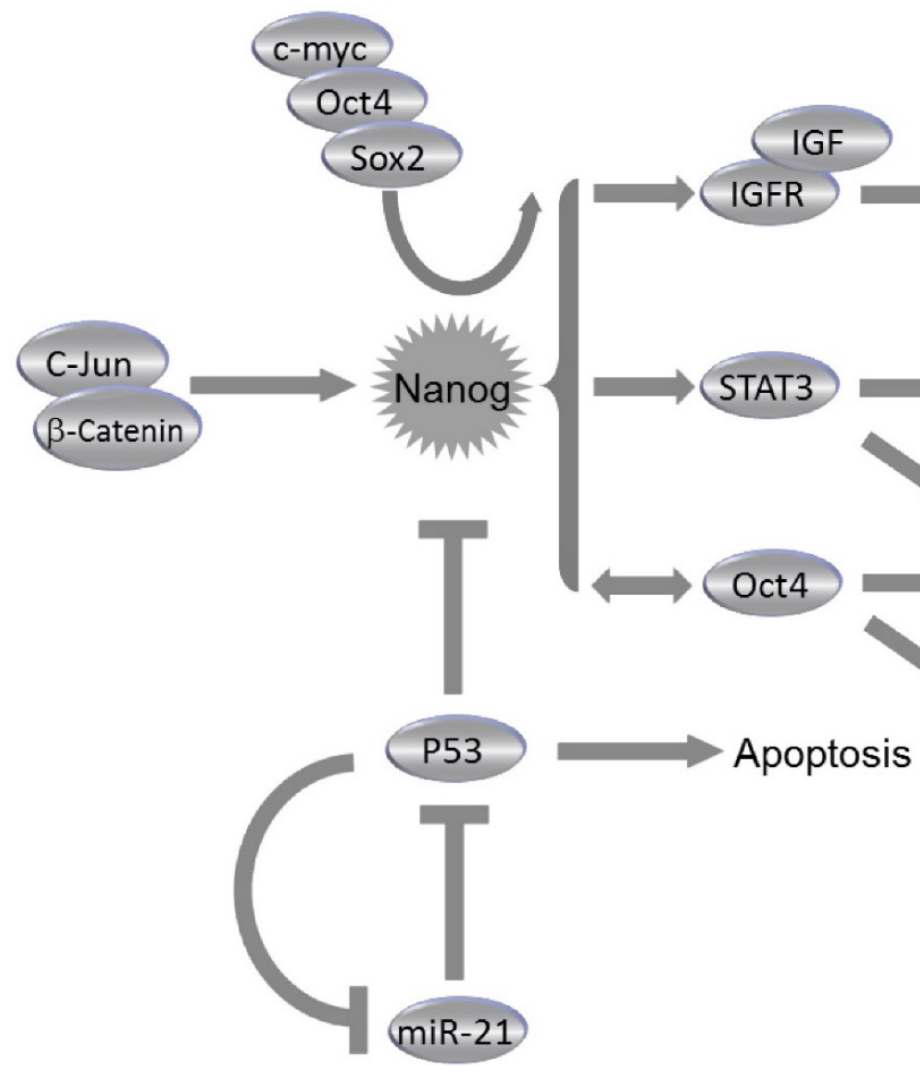

Figure 3. Roles of Nanog in Cancer. Collaborating with c-myc, Oct4 and Sox2, Nanog regulates self-renewal, proliferation, epithelial-mesenchymal transition (EMT) and metastasis of cancer cells through miscellaneous pathways. Nanog expression is regulated by c-Jun/ $\beta$-catenin and can be suppressed by $\mathrm{p} 53$. Consistently, miR-21 can activate Nanog pathway by direct inhibition of p53. 


\section{Glioblastoma multiforme}

Glioblastoma multiforme (GBM) is a highly invasive brain tumor with very high mortality rate $^{49,50}$. Hedgehog-GLI (HH-GLI) signaling pathway is found as an important signaling pathway in GBM, which is responsible for GBM cell growth, CSC expansion and specific expression of ESC-like stemness signature ${ }^{50,51}$. Furthermore, Hedgehog pathway was demonstrated to promote metastatic capacity and CSC expansion through direct regulation of Nanog, which has proved to be a novel HH-GLI mediator essential for GBM formation 50,51 . The function of Nanog is conserved, since GBM cell growth and $\mathrm{CD} 133^{+}$GBM CSC expansion rely on Nanog expression ${ }^{49,51}$. Tissue microarrays of 80 low-grade (WHO Grade II) and 98 high-grade human gliomas (WHO Grades III and IV) revealed upregulated protein levels of Nanog, Klf4, Oct4 and Sox2 in high-grade gliomas, as compared to low-grade ones ${ }^{52}$. In the multivariate model, Nanog was identified as an independent prognostic factor in the subgroups of low-grade astrocytoma, high-grade astrocytoma and glioblastomas only ${ }^{52}$.

\section{Colorectal cancers}

Nanog is found to be expressed in the form of both Nanog1 and NanogP8 in human colorectal cancer $(\mathrm{CRC})^{53}$. Clonogenic potential and tumor formation capacity of CSCs in CRC could be enhanced by increased expression of Nanog153,54. In CRC patients, Nanog1 is expressed only in a small fraction of cancer cells. However, a single Nanog1+ CRC cell could form a spherical aggregate, similar to the embryoid body derived from ESCs ${ }^{53}$. Nanog1 expression is regulated by c-Jun and $\beta$-catenin/transcription factor 4 complex. Ectopic expression of c-Jun in murine ESCs results in the development of larger xenograft tumors with a higher cell density compared to controls. Chromatin immunoprecipitation assays demonstrate that c-Jun binds to the Nanog1 promoter via the octamer M1 DNA element ${ }^{53-55}$. Moreover, reported evidence suggests that Nanog1 can induce CSC to adopt a stem-like phenotype and contribute to CSC pool expansion in $\mathrm{CRC}^{54,55}$.

On the other hand, immunohistochemistry analysis of 175 colorectal tumor samples revealed that overexpression of NanogP8 was strongly correlated with poor prognosis, lymph node metastasis and Dukes classification of CRC patients ${ }^{56}$. A study of NanogP8 expression level in $\mathrm{CD}_{133^{+}}$and CD133tumor cells of CRC indicated that NanogP8 expression was significantly higher in $\mathrm{CD} 133^{+}$tumor cells compared to CD133- ones. NanogP8 is expressed in 72 of 360 cases of $\mathrm{CRC}^{57}$, and is associated with histological grade, lymph node metastasis, TNM stage, and liver metastasis ${ }^{57}$. Heretofore, expression level of NanogP8 is considered as a significant biomarker for prediction of postoperative liver metastasis of CRC patients.

\section{Gastric adenocarcinoma}

In clinical samples of 105 patients undergoing retrospective surgery for gastric adenocarcinoma, the expression of Nanog protein level is significantly higher in cancer tissues compared to that in surrounding non-tumor tissues ${ }^{58}$. Overexpression of Nanog protein was positively correlated with lymph node status, infiltration scope and differentiation stages of gastric cancer, as well as advanced clinical stages of patients ${ }^{58,59}$. Another study discovered that the expression of Nanog (Nanog1 and NanogP8) was much higher in tumor than that in para-tumor tissues, and its overall level is correlated with poor prognosis ${ }^{59}$. Interestingly, Helicobacter pylori-infected cancer cells exhibited CSC-like properties, including an increased expression level of CSC-specific surface markers, including CD44 and Lgr5, as well as Nanog, Oct4 and c-Myc, and an enhanced capacity for self-renewal ${ }^{59}$. Knockdown of Nanog by RNAi in gastric cancer cell lines was shown to inhibit cell proliferation, and promote cell apoptosis and S-phase cell cycle arrest ${ }^{43}$.

\section{Oral squamous cell carcinoma}

Oral squamous cell carcinoma (OSCC) is a very aggressive cancer with high mortality rates, and patients usually die of cancer or the complications within 5 years upon initial diagnosis ${ }^{60}$. Nanog is found to regulate drug resistance, which is a challenging issue in treatment of OSCC. Cisplatin-resistant OSCC cells expressed high levels of stemness markers (Nanog, Oct4, Bmi-1, CD117, CD133 and ABCG2) and have much higher sphere-forming/self-renewal capability, as compared with cisplatin-sensitive ones ${ }^{60}$. Cisplatin-resistant OSCC cells also exhibited enhanced ability in migration, invasion, and clonogenicity ${ }^{60}$. Consistent with these in vitro results, expression levels of Oct4 and Nanog were significantly upregulated in cisplatin-resistant OSCC patients ${ }^{60}$. Recently, a retrospective study involving 60 cases of OSCC revealed that Nanog expression was identified in 52 cases $(86.7 \%)$ and its expression was higher in primary foci of poorly differentiated OSCC than well-differentiated OSCC tissue ${ }^{61}$. Moreover, the level of Nanog was even higher in metastatic foci as compared with primary foci, and Nanog expression in metastatic foci was maintained at high level following 
preoperative adjuvant therapy, suggesting Nanog-expressing OSCC cells tend to metastasize, and may be resistant to preoperative adjuvant therapy ${ }^{61}$. In clinical settings, the expression pattern of stemness-related transcriptional factors, $\beta$-catenin, Oct4, and Nanog, exhibited significantly positive correlation with lymph node metastasis, advanced cancer stages, and worse prognosis in OSCC patients ${ }^{60,61}$. Taken together, Nanog was found to be involved in OSCC initiation, carcinogenesis and chemoresistance to oral cancer therapy by enhancing CSC activities, which included increased expression levels of drug efflux pumps, alteration in programmed cell death and cell cycle, and reinforced DNA repair capacity ${ }^{61,62}$.

\section{Breast cancer}

Nanog is overexpressed in breast cancer as well ${ }^{63}$. An inducible Nanog transgenic mouse model has elucidated the role of Nanog in breast carcinogenesis. Induction of Nanog in the mammary gland was not sufficient to induce mammary tumor. However, when Nanog was co-expressed with Wnt-1 in the mouse mammary gland, mammary tumorigenesis and metastasis were observed ${ }^{63}$. Microarray analysis showed that the ectopic expression of Nanog deregulated the expression of numerous genes associated with tumorigenesis and metastasis, proposing a correlation between Nanog expression and poor prognosis of human breast cancer patients ${ }^{63}$. In a separate study enrolling 100 breast cancer patients, patients with strong expression of Nanog had significantly lower disease-free survival rates and overall survival rates than those with weak expression of Nanog ${ }^{64}$.

\section{Pancreatic cancer}

Pancreatic cancer is notorious for difficult diagnosis at early stage and poor recurrence-free prognosis. Nanog and Oct4 are regarded as possible biomarkers in auxiliary diagnosis of pancreatic cancer ${ }^{65}$. The pancreatic CSCs isolated from PANC-1 cell line by flow cytometry exhibited higher expression levels of Nanog and Oct4, which were collaboratively demonstrated to predict a worse prognosis in pancreatic cancer patients ${ }^{65}$. Double knockdown of Nanog and Oct4 significantly reduced proliferation, migration, invasion, chemoresistance, and tumor regeneration capacity of pancreatic CSCs in vitro and in vivo, suggesting Nanog and Oct 4 may serve as potential indicators for prognosis and novel therapeutic targets for pancreatic cancer 65 . In addition, Amsterdam et al reported that LGR5 and Nanog were co-expressed in clusters of beta cells in normal pancreas, as well as in cancerous pancreas ${ }^{66}$. Their study indicated that the islet's beta cells, which express LGR5 and Nanog markers, may be the initiating cells of pancreatic cancer, which can migrate from the islets to form the ductal cancerous tissue, probably after mutation and de-differentiation ${ }^{66}$.

\section{Ovarian cancer}

Ovarian cancer is the most lethal in all gynecological malignancies, and the identification of novel prognostic indicators and therapeutic targets for ovarian cancer is crucial 3,67 . The expression of Nanog in normal ovarian tissue, benign, borderline, and malignant tumors showed a gradually rising trend ${ }^{68}$. Furthermore, Nanog expression level was closely related with tumor stages, and it was expressed in almost all stage IV patients ${ }^{68}$. Exactly, Nanog is overexpressed in the nuclei of cancer tissue, and increased nuclear Nanog expression was significantly associated with high-grade cancer development, severe histological subtypes, reduced chemosensitivity, poor overall and disease-free survival ${ }^{67,68}$. Stable knockdown of Nanog impeded ovarian cancer cell proliferation, migration and invasion, accompanied by an increased mRNA expression of E-cadherin, caveolin-1, FOXO1, FOXO3a, FOXJ1 and FOXB1. Consistently, ectopic Nanog overexpression enhanced ovarian cancer cell migration and invasion capacity along with decreased mRNA levels of the aforementioned factors ${ }^{3,23}$.

\section{Lung cancer}

In a retrospective study of 163 lung cancer patients, the level of Nanog protein in lung cancer tissues was found to be higher as compared with their normal counterparts, and was positively correlated with tumor stages ${ }^{69}$. Not surprisingly, survival analyses showed overexpression of Nanog protein predicted a worse prognosis for lung cancer patients ${ }^{69}$. In another study, Nanog mRNA was detected in $84.8 \%$ (39 out of 46) of lung cancer tissue samples. The sensitivity and specificity of the Nanog mRNA-based diagnostic technique was 80.4 and 93.3\%, respectively ${ }^{70}$. Actually, Nanog mRNA was highly expressed even in the early clinical stages of lung cancer, suggesting that Nanog mRNA detection might be a novel method to confirm the diagnosis of lung cancer, irrespective of the clinical stage ${ }^{70}$.

\section{Conclusion}

As a vital transcription factor that maintains self-renewal and pluripotency of ESCs, Nanog is also expressed in many types of cancers and its expression is pivotal to confer CSC-like phenotype. Increased expression level of Nanog is bound up with drug resistance, metastasis and poor postoperative 
prognosis. Manipulation on Nanog expression at transcription level or via post-transcriptional regulation methods has shown promising therapeutic effects in different cancer models. Heretofore, Nanog was found to be involved in multiple oncogenic signal transduction pathways, including chemoresistance, EMT, metastasis, and CSC expansion. However, currently most of the mechanistic studies were based on ESCs or CSCs from human or mouse under in vitro circumstances, and the system biology approaches might be needed to allow deeper understanding of the temporal and intensity dynamics of Nanog regulatory network in ESCs, tumor cells and CSCs. Meanwhile, the roles of Nanog in cancer prognosis and anti-cancer therapeutics have become another hotspot. Recent findings have demonstrated that Nanog may act as a critical therapy target in clinical settings, as well as a biomarker for the diagnosis, and assessment indicator for prognosis and anti-cancer therapeutic efficacy.

\section{Acknowledgement}

The work was financially supported by National Natural Science Foundation of China (grant No. $81372750 \& 81572518$ ) and Science \& Technology Development Fund of Shanghai Pudong (grant No. PKJ2014-Y10) to TY, and The Key Basic Applied Project of Hebei Provincial Department of Science \& Technology (grant No. 15967730D) to WZ.

\section{Competing Interests}

The authors have declared that no competing interest exists.

\section{References}

1. Mitsui K, Tokuzawa $Y$, Itoh $\mathrm{H}$, et al. The homeoprotein Nanog is required for maintenance of pluripotency in mouse epiblast and ES cells. Cell. 2003; 113: 631-642.

2. Chambers I, Colby D, Robertson M, et al. Functional expression cloning of Nanog, a pluripotency sustaining factor in embryonic stem cells. Cell. 2003; 113: 643-655.

3. Siu MK, Wong ES, Kong DS, et al. Stem cell transcription factor NANOG controls cell migration and invasion via dysregulation of E-cadherin and FoxJ1 and contributes to adverse clinical outcome in ovarian cancers. Oncogene. 2013; 32: 3500-3509.

4. Torres-Padilla ME, Chambers I. Transcription factor heterogeneity in pluripotent stem cells: a stochastic advantage. Development. 2014; 141: 2173-2181.

5. Mimeault M, Batra SK. Frequent gene products and molecular pathways altered in prostate cancer- and metastasis-initiating cells and their progenies and novel promising multitargeted therapies. Mol Med. 2011; 17: 949-964.

6. Saunders A, Faiola F, Wang J. Concise review: pursuing self-renewal and pluripotency with the stem cell factor Nanog. Stem Cells. 2013; 31: 1227-1236.

7. Nakai-Futatsugi $Y$, Niwa H. Transcription factor network in embryonic stem cells: heterogeneity under the stringency. Biol Pharm Bull. 2013; 36: 166-170.

8. Chang DF, Tsai SC, Wang XC, et al. Molecular characterization of the human NANOG protein. Stem Cells. 2009; 27: 812-821.

9. Eberle I, Pless B, Braun $\mathrm{M}$, et al. Transcriptional properties of human NANOG1 and NANOG2 in acute leukemic cells. Nucleic Acids Res. 2010; 38 : 5384-5395.

10. Scerbo P, Markov GV, Vivien C, et al. On the origin and evolutionary history of NANOG. PLoS One. 2014; 9: e85104.

11. Fairbanks DJ, Maughan PJ. Evolution of the NANOG pseudogene family in the human and chimpanzee genomes. BMC Evol Biol. 2006; 6: 12.

12. Badeaux MA, Jeter $C R$, Gong $S$, et al. In vivo functional studies of tumor-specific retrogene NanogP8 in transgenic animals. Cell Cycle. 2013; 12: 2395-2408.
13. Ambady S, Malcuit C, Kashpur O, et al. Expression of NANOG and NANOGP8 in a variety of undifferentiated and differentiated human cells. Int J Dev Biol. 2010; 54: 1743-1754.

14. Palla AR, Piazzolla D, Abad M, et al. Reprogramming activity of NANOGP8, a NANOG family member widely expressed in cancer. Oncogene. 2014; 33: 2513-2519.

15. Ding $\mathrm{Y}, \mathrm{Yu}$ AQ, Wang $\mathrm{XL}$, et al. Forced expression of Nanog with mRNA synthesized in vitro to evaluate the malignancy of HeLa cells through acquiring cancer stem cell phenotypes. Oncol Rep. 2016; 35: 2643-2650.

16. Yong X, Tang B, Xiao YF, et al. Helicobacter pylori upregulates Nanog and Oct4 via Wnt/ $\beta$-catenin signaling pathway to promote cancer stem cell-like properties in human gastric cancer. Cancer Lett. 2016; 374: 292-303.

17. Yu AQ, Ding Y, Li CL, et al. TALEN-induced disruption of Nanog expression results in reduced proliferation, invasiveness and migration, increased chemosensitivity and reversal of EMT in HepG2 cells. Oncol Rep. 2016; 35: 1657-1663.

18. $\mathrm{Hu} \mathrm{C}, \mathrm{Xu} \mathrm{L}$, Liang $\mathrm{S}$, et al. Lentivirus-mediated shRNA targeting Nanog inhibits cell proliferation and attenuates cancer stem cell activities in breast cancer. J Drug Target. 2016; 24: 422-432.

19. Kawamura N, Nimura $\mathrm{K}$, Nagano $\mathrm{H}$, et al. CRISPR/Cas9-mediated gene knockout of NANOG and NANOGP8 decreases the malignant potential of prostate cancer cells. Oncotarget. 2015; 6: 22361-22374.

20. Hart AH, Hartley L, Ibrahim M, et al. Identification, cloning and expression analysis of the pluripotency promoting Nanog genes in mouse and human. Dev Dyn. 2004; 230: 187-198.

21. Niwa H, Burdon T, Chambers I, et al. Self-renewal of pluripotent embryonic stem cells is mediated via activation of STAT3. Genes Dev. 1998; 12: 2048-2060.

22. Mullin NP, Yates A, Rowe AJ, et al. The pluripotency rheostat Nanog functions as a dimer. Biochem J. 2008; 411: 227-231.

23. Xu CX, Xu M, Tan L, et al. MicroRNA miR-214 regulates ovarian cancer cell stemness by targeting p53/Nanog. J Biol Chem. 2012; 287: 34970-34978.

24. Wang Z, Ma T, Chi X, et al. Aromatic residues in the C-terminal domain 2 are required for Nanog to mediate LIF-independent self-renewal of mouse embryonic stem cells. J Biol Chem. 2008; 283: 4480-4489.

25. Wang J, Levasseur DN, Orkin SH. Requirement of Nanog dimerization for stem cell self-renewal and pluripotency. Proc Natl Acad Sci U S A. 2008; 105: 6326-6331.

26. dos Santos RL, Tosti L, Radzisheuskaya A, et al. MBD3/NuRD facilitates induction of pluripotency in a context-dependent manner. Cell Stem Cell. 2014; 15: 102-110.

27. Liang J, Wan M, Zhang $\mathrm{Y}$, et al. Nanog and Oct4 associate with unique transcriptional repression complexes in embryonic stem cells. Nat Cell Biol. 2008; 10: 731-739.

28. Zhang X, Neganova I, Przyborski S, et al. A role for NANOG in G1 to S transition in human embryonic stem cells through direct binding of CDK6 and CDC25A. J Cell Biol. 2009; 184: 67-82.

29. Cao J, Li L, Chen C, et al. RNA interference-mediated silencing of NANOG leads to reduced proliferation and self-renewal, cell cycle arrest and apoptosis in T-cell acute lymphoblastic leukemia cells via the p53 signaling pathway. Leuk Res. 2013; 37: 1170-1177.

30. Han J, Zhang F, Yu M, et al. RNA interference-mediated silencing of NANOG reduces cell proliferation and induces G0/G1 cell cycle arrest in breast cancer cells. Cancer Lett. 2012; 321: 80-88.

31. Lim LS, Hong FH, Kunarso G, et al. The pluripotency regulator Zic3 is a direct activator of the Nanog promoter in ESCs. Stem Cells. 2010; 28: 1961-1969.

32. Lee K, Hamm J, Whitworth K, et al. Dynamics of TET family expression in porcine preimplantation embryos is related to zygotic genome activation and required for the maintenance of NANOG. Dev Biol. 2014; 386: 86-95.

33. Costa Y, Ding J, Theunissen TW, et al. NANOG-dependent function of TET1 and TET2 in establishment of pluripotency. Nature. 2013; 495: 370-374.

34. Chiou SH, Wang ML, Chou YT, et al. Coexpression of Oct4 and Nanog enhances malignancy in lung adenocarcinoma by inducing cancer stem cell-like properties and epithelial-mesenchymal transdifferentiation. Cancer Res. 2010; 70: 10433-10444.

35. Chen L, Kasai T, Li Y, et al. A model of cancer stem cells derived from mouse induced pluripotent stem cells. PLoS One. 2012; 7: e33544.

36. Xie X, Piao L, Cavey GS, et al. Phosphorylation of Nanog is essential to regulate Bmi1 and promote tumorigenesis. Oncogene. 2014; 33: 2040-2052.

37. Tommasi S, Zheng A, Yoon JI, et al. Epigenetic targeting of the Nanog pathway and signaling networks during chemical carcinogenesis. Carcinogenesis. 2014; 35: 1726-1736.

38. Sun C, Sun L, Jiang K, et al. NANOG promotes liver cancer cell invasion by inducing epithelial-mesenchymal transition through NODAL/SMAD3 signaling pathway. Int J Biochem Cell Biol. 2013; 45: 1099-1108.

39. Jeter CR, Badeaux M, Choy G, et al. Functional evidence that the self-renewal gene NANOG regulates human tumor development. Stem Cells. 2009; 27: 993-1005.

40. Li Y, Higashiyama S, Shimakage M, et al. Involvement of NANOG upregulation in malignant progression of human cells. DNA Cell Biol. 2013; 32: $104-110$

41. Choi SC, Choi JH, Park CY, et al. Nanog regulates molecules involved in stemness and cell cycle-signaling pathway for maintenance of pluripotency of P19 embryonal carcinoma stem cells. J Cell Physiol. 2012; 227: 3678-3692. 
42. Jeter CR, Liu B, Liu X, et al. NANOG promotes cancer stem cell characteristics and prostate cancer resistance to androgen deprivation. Oncogene. 2011; 30: 3833-3845.

43. Ji W, Jiang Z. Effect of shRNA-mediated inhibition of Nanog gene expression on the behavior of human gastric cancer cells. Oncol Lett. 2013; 6: 367-374.

44. Sato A, Okada M, Shibuya K, et al. Resveratrol promotes proteasome-dependent degradation of Nanog via p53 activation and induces differentiation of glioma stem cells. Stem Cell Res. 2013; 11: 601-610.

45. Zhang K, Fowler M, Glass J, et al. Activated 5'flanking region of NANOGP8 in a self-renewal environment is associated with increased sphere formation and tumor growth of prostate cancer cells. Prostate. 2014; 74: 381-394.

46. Miyazawa K, Tanaka T, Nakai D, et al. Immunohistochemical expression of four different stem cell markers in prostate cancer: High expression of NANOG in conjunction with hypoxia-inducible factor-1a expression is involved in prostate epithelial malignancy. Oncol Lett. 2014; 8: 985-992.

47. Yin $\mathrm{X}, \mathrm{Li} \mathrm{YW}$, Zhang BH, et al. Coexpression of stemness factors Oct4 and Nanog predict liver resection. Ann Surg Oncol. 2012; 19: 2877-2887.

48. Shan J, Shen J, Liu L, et al. Nanog regulates self-renewal of cancer stem cells through the insulin-like growth factor pathway in human hepatocellular carcinoma. Hepatology. 2012; 56: 1004-1014.

49. Higgins DM, Wang R, Milligan B, et al. Brain tumor stem cell multipotency correlates with nanog expression and extent of passaging in human glioblastoma xenografts. Oncotarget. 2013; 4: 792-801.

50. Moon JH, Kwon S, Jun EK, et al. Nanog-induced dedifferentiation of p53-deficient mouse astrocytes into brain cancer stem-like cells. Biochem Biophys Res Commun. 2011; 412: 175-181.

51. Zbinden M, Duquet A, Lorente-Trigos A, et al. NANOG regulates glioma stem cells and is essential in vivo acting in a cross-functional network with GLI1 and p53. EMBO J. 2010; 29: 2659-2674.

52. Elsir T, Edqvist PH, Carlson J, et al. A study of embryonic stem cell-related proteins in human astrocytomas: identification of Nanog as a predictor of survival. Int J Cancer. 2014; 134: 1123-1131.

53. Ibrahim EE, Babaei-Jadidi R, Saadeddin A, et al. Embryonic NANOG activity defines colorectal cancer stem cells and modulates through AP1- and TCF-dependent mechanisms. Stem Cells. 2012; 30: 2076-2087.

54. Zhang J, Espinoza LA, Kinders RJ, et al. NANOG modulates stemness in human colorectal cancer. Oncogene. 2013; 32: 4397-4405.

55. Ishiguro $\mathrm{T}$, Sato $\mathrm{A}$, Ohata $\mathrm{H}$, et al. Differential expression of nanog1 and nanogp8 in colon cancer cells. Biochem Biophys Res Commun. 2012; 418: 199-204.

56. Meng HM, Zheng $\mathrm{P}$, Wang $X Y$, et al. Over-expression of Nanog predicts tumor progression and poor prognosis in colorectal cancer. Cancer Biol Ther. 2010; 9: 295-302.

57. Xu F, Dai C, Zhang R, et al. Nanog: a potential biomarker for liver metastasis of colorectal cancer. Dig Dis Sci. 2012; 57: 2340-2346.

58. Lin T, Ding YQ, Li JM. Overexpression of Nanog protein is associated with poor prognosis in gastric adenocarcinoma. Med Oncol. 2012; 29: 878-885.

59. Zhao X, Wang F, Hou M. Expression of stem cell markers nanog and PSCA in gastric cancer and its significance. Oncol Lett. 2016; 11: 442-448.

60. Tsai LL, Yu CC, Chang YC, et al. Markedly increased Oct4 and Nanog expression correlates with cisplatin resistance in oral squamous cell carcinoma. J Oral Pathol Med. 2011; 40: 621-628.

61. Watanabe M, Ohnishi $Y$, Inoue $H$, et al. NANOG expression correlates with differentiation, metastasis and resistance to preoperative adjuvant therapy in oral squamous cell carcinoma. Oncol Lett. 2014; 7: 35-40.

62. Sinha N, Mukhopadhyay S, Das DN, et al. Relevance of cancer initiating/stem cells in carcinogenesis and therapy resistance in oral cancer. Oral Oncol. 2013; 49: 854-862.

63. Lu X, Mazur SJ, Lin T, et al. The pluripotency factor nanog promotes breast cancer tumorigenesis and metastasis. Oncogene. 2014; 33: 2655-2664.

64. Nagata T, Shimada $Y$, Sekine $S$, et al. Prognostic significance of NANOG and KLF4 for breast cancer. Breast Cancer. 2014; 21: 96-101.

65. Lu Y, Zhu H, Shan H, et al. Knockdown of Oct4 and Nanog expression inhibits the stemness of pancreatic cancer cells. Cancer Lett. 2013; 340: 113-123.

66. Amsterdam A, Raanan C, Schreiber L, et al. LGR5 and Nanog identify stem cell signature of pancreas beta cells which initiate pancreatic cancer. Biochem Biophys Res Commun. 2013; 433: 157-162.

67. Zhang S, Balch $\mathrm{C}$, Chan MW, et al. Identification and characterization of ovarian cancer-initiating cells from primary human tumors. Cancer Res. 2008; 68: 4311-4320.

68. Pan Y, Jiao J, Zhou C, et al. Nanog is highly expressed in ovarian serous cystadenocarcinoma and correlated with clinical stage and pathological grade. Pathobiology. 2010; 77: 283-288.

69. Du Y, Ma C, Wang Z, et al. Nanog, a novel prognostic marker for lung cancer. Surg Oncol. 2013; 22: 224-229.

70. Nirasawa S, Kobayashi D, Tsuji N, et al. Diagnostic relevance of overexpressed Nanog gene in early lung cancers. Oncol Rep. 2009; 22: 587-591. 